\title{
Further development in the assessment of psychological flexibility: validation of the German committed action questionnaire
}

\author{
Yannik Terhorst ${ }^{1,2^{*}}$ (D), Harald Baumeister ${ }^{2}$, Lance M. McCracken ${ }^{3}$ and Jiaxi Lin ${ }^{4}$
}

\begin{abstract}
Background: Psychological flexibility is considered a fundamental aspect of health. It includes six interrelated facets: 1) cognitive defusion, 2) acceptance, 3) contact with the present moment, 4) self-as-context, 5) values, and 6) committed action. To gain further insight into psychological flexibility and its effects on health, reliable and valid instruments to assess all facets are needed. Committed action is one facet that is understudied. A long and short version of a validated measure (CAQ and CAQ-8) have been developed in English. Currently, there are no German versions of the CAQ. Aim of this study is to validate German-language versions of these in a chronic pain population.

Methods: The CAQ instructions and items were translated and evaluated in a chronic pain population ( $N=181)$. Confirmatory factor analysis and Mokken scale analysis were conducted to evaluate the German questionnaires. Correlations with health outcomes, including quality of life (SF-12), physical and emotional functioning (MPI, BPI, PHQ-9, GAD-7), pain intensity, and with other facets of psychological flexibility (CPAQ, FAH-II) were investigated for convergent validity purposes. Scale reliability was assessed by the alpha, MS, lambda-2, LCRC, and omega coefficient.
\end{abstract}

Results: A bifactor model consisting of one general factor and two methodological factors emerged from the analysis. Criteria for reliability and validity were met. Medium to strong correlations to health outcomes and other facets of psychological flexibility were found. Results were similar to the original English version.

Conclusions: The present study presents a valid and reliable instrument to investigate committed action in German populations. Future studies could expand the present findings by evaluating the German CAQ versions in non-pain populations. The role of committed action and the wider psychological flexibility model in pain and other conditions deserves further investigation.

Keywords: Committed action, Psychological flexibility, Chronic pain

\footnotetext{
*Correspondence: yannik.terhorst@uni-ulm.de

'Department of Research Methods, Institute of Psychology and Education,

Ulm University, Albert-Einstein-Allee 47, 89069 Ulm, Germany

2Department of Clinical Psychology and Psychotherapy, Institute of

Psychology and Education, Ulm University, Lise-Meitner-Str. 16, 89081 Ulm, Germany

Full list of author information is available at the end of the article
}

(c) The Author(s). 2020 Open Access This article is licensed under a Creative Commons Attribution 4.0 International License, which permits use, sharing, adaptation, distribution and reproduction in any medium or format, as long as you give appropriate credit to the original author(s) and the source, provide a link to the Creative Commons licence, and indicate if changes were made. The images or other third party material in this article are included in the article's Creative Commons licence, unless indicated otherwise in a credit line to the material. If material is not included in the article's Creative Commons licence and your intended use is not permitted by statutory regulation or exceeds the permitted use, you will need to obtain permission directly from the copyright holder. To view a copy of this licence, visit http://creativecommons.org/licenses/by/4.0/ The Creative Commons Public Domain Dedication waiver (http://creativecommons.org/publicdomain/zero/1.0/) applies to the data made available in this article, unless otherwise stated in a credit line to the data. 


\section{Background}

Psychological flexibility is considered a fundamental aspect of health [1], and is defined as an ability to contact the present moment more fully as conscious human and to persist in or change behavior in accordance with one's goals, values and what the situation's affords [2]. In total, psychological flexibility consists of six interrelated facets: cognitive defusion, acceptance, contact with the present moment, self-as-context, values, and committed action [2]. The psychological flexibility model focuses on patterns of actions or behavior and has been identified as a key factor in many disorders and conditions (e.g. chronic pain, somatization, depression, anxiety, or psychological distress) [1-6].

Therapies such as Acceptance and Commitment Therapy (ACT) that focus on psychological flexibility have proven to be effective across several mental and somatic conditions (e.g. depression, anxiety and pain disorders) and settings (e.g. face-to-face, internet-based interventions); for reviews see [7-10]. However, in order to identify effective therapy components for specific patient populations as well as to better understand how therapy works [5, 11-14] it is important that key therapeutic processes of change can be measured reliably. Thus, measures have been developed or adopted to reflect psychological flexibility and its facets, including acceptance [15], present-focused awareness [16], cognitive defusion [17], self as context [18, 19] and values [20]. Several facets of psychological flexibility are well studied [5, 2124]. However, the facet committed actions has not been investigated extensively [25].

Committed action is action guided by one's goals and values, which is persistent in that it can incorporate discomfort and failure, and is flexible in that it can be stopped if it is unsuccessful [25, 26]. McCracken and colleagues developed a measure called Committed Actions Questionnaire (CAQ) [25] and a short version of this questionnaire CAQ-8 [26] in English to assess committed action. Both the long and the short version of the CAQ show satisfactory reliability and validity [25, 26]. To make the assessment of committed action widely accessible, translations and validations in other languages are needed. While the CAQ- 8 provides a shorter and psychometrical sound assessment of committed action, a shorter item inventory is also accompanied by less content and potentially less information and details about the underlying behavior patterns [26]. Thus, validation of CAQ and CAQ-8 is needed. Currently, there are no German versions of the CAQ and CAQ-8.

The aim of this study is to validate German-language versions of the original version of the CAQ and the short version (CAQ-8) in a chronic pain population. The study examines the reliability and validity of both versions. In addition, based on item response theory (IRT),
Mokken scaling analysis (MSA) will be applied to examine the dimensionality of the questionnaires and to determine whether the use of sum scores is adequate. Furthermore, associations between committed action and 1) the facet acceptance, 2) pain interference, 3) depression, 4) anxiety, 5) pain intensity, and 6) quality of life are investigated.

Construct validity of the original CAQ was supported by a latent two-factor and bifactor model, which fitted the data best [26]. We assume this to be equivalent for the CAQ-G. In terms of convergent and discriminate validity there is strong evidence for associations between committed action, mental health, pain-related symptoms, and other facets of psychological flexibility [2532]: Based on these findings we expect medium positive correlations with health and medium to strong positive correlations to other facets of psychological flexibility.

\section{Methods \\ Sample}

The present sample consists of a subsample of individuals with chronic pain who participated in a threearmed randomized controlled trial (RCT) on the effectiveness of an online ACT based intervention called ACTonPain [33, 34]. Inclusion and exclusion criteria and the study procedure of ACTonPain are described in detail elsewhere [33, 34]. All participants gave informed consent for the use of their data for research purposes. The ACTonPain study was approved by the ethics committee of University Freiburg and registered at the German Clinical Trial Register (DRKS): DRKS00006183.

181 participants completed the CAQ-G at the 6 months follow-up of the ACTonPain study. Thus, $N=$ 181 participants were included in the analyses. Participants were mainly women (86\%). Average age was 51.97 years $(\mathrm{SD}=13.12)$. The majority was employed $(58 \%)$ and married (55\%). Further descriptive characteristics of this subsample are summarized in Table 1.

\section{Translation of the CAQ}

We followed international recommendations for translating and back-translating the CAQ [35]. First, the CAQ was translated from the original to German by a German clinical psychologist (JL) fluent in English and with specific knowledge of the research area. The questionnaire was then back-translated by two Germans, one psychologist fluent in English and with experience in working with instrument validation (HB) and another who worked as a translator for both directions (PS). Thereafter, the translated and back-translated versions were evaluated by the author of the English language original (LM). Discrepancies were discussed and resolved by consensus. 
Table 1 Descriptive characteristics $(N=181)$

\begin{tabular}{|c|c|}
\hline \multicolumn{2}{|l|}{ Variable } \\
\hline Gender & $86 \%$ female \\
\hline Age & Mean $(M)=51.97(s d=13.12)$ years \\
\hline \multirow[t]{6}{*}{ Family status } & Single $17 \%$ \\
\hline & In relationship 15\% \\
\hline & Married 55\% \\
\hline & Divorced 7\% \\
\hline & Living separated $2 \%$ \\
\hline & Widowed 4\% \\
\hline \multirow[t]{4}{*}{ Education } & Basic school qualification 14\% \\
\hline & Secondary school qualification 36\% \\
\hline & Technical college qualification 18\% \\
\hline & College qualification and higher $31 \%$ \\
\hline \multirow[t]{4}{*}{ Employment } & Unemployed 4\% \\
\hline & In education or study $3 \%$ \\
\hline & Employed 58\% \\
\hline & Retired 35\% \\
\hline \multirow[t]{2}{*}{ Pain duration in weeks } & Median (MD) = 60 (95\% Cl: 9-463) \\
\hline & $M=110$ \\
\hline \multirow[t]{5}{*}{ Pain location } & Head 14\% \\
\hline & Neck 11\% \\
\hline & Shoulder $6 \%$ \\
\hline & Back 62\% \\
\hline & Other 35\% \\
\hline
\end{tabular}

The translated versions of the CAQ-G and CAQ-8-G are presented in the additional file 1.

\section{Measures}

\section{German committed action questionnaire}

The German version of the committed action questionnaire (CAQ-G) is a translation of the English CAQ [25]. The 18-item version contains nine positive keyed items and nine negative keyed items. Responders have to indicate to which extent an item applies to them on a scale from 0 (never true) to 6 (always true). The reliability of the 18 item version was high $(\alpha=.91)$ [25]. Further correlation analyses in previous studies showed good convergent and divergent validity with other constructs (e.g. pain acceptance or emotional functionality) [25]. Items of the CAQ were reduced to eight for a short version [26]. Reliability of the CAQ-8 is good $(\alpha=.87)$ [26].

\section{Physical and emotional functioning}

In accordance with the Initiative on Methods, Measurement, and Pain Assessment in Clinical Trials (IMMP $\mathrm{ACT})$ recommendations [36, 37] the German Multidimensional Pain Inventory (MPI) and the German Brief Pain Inventory (BPI) were used to assess pain interference. The German MPI consists of 10 questions assessing interference on a behavioral (e.g. daily activities) and emotional level (e.g. ability to experience joy) with an excellent reliability $(\alpha=.94)$ [38]. Interference in physical functioning (e.g. walking ability), emotional functioning (e.g. mood) and sleep is assessed with seven items in the German BPI. Reliability of the German BPI is excellent with a reliability of $\alpha=.88$ [39].

Depression was assessed with the German Patient Health Questionnaire (PHQ-9) [40]. The German Generalized Anxiety Disorder questionnaire (GAD-7) was used to assess anxiety [41, 42]. Both, the German PHQ-9 and the German GAD-7 show an excellent reliability of $\alpha=.89[41,43]$.

\section{Health related quality of life}

To assess health-related quality of life the Short Form 12 (SF12) was used [44]. The SF-12 is divided into physical (SF12 PCS) and mental health (SF12 MCS) and covers eight health domains: physical functioning, role limitations, pain, general health perception, vitality, mental health, emotional role and social functioning [44]. The physical subscale $(\alpha=.77)$ and mental health subscale $(\alpha=.80)$ have good reliability [44].

\section{Pain intensity}

As recommended by the IMMPACT pain intensity was assessed with a 11 point numerical rating scale (NRS), on which responders indicated average pain during the last week from 0 to 10 , with $0=$ "no pain" and $10=$ "pain as bad as you can imagine" [36, 37].

\section{Psychological flexibility}

To assess psychological flexibility, the German version of the Acceptance and Action Questionnaire - II (AAQ-II [45] German: Fragebogen zu Akzeptanz und Handeln II (FAH-II [46]) was used. This questionnaire consists of 7 items and shows good to excellent psychometric properties in a German sample [46]. On a 7point scale that ranges from $0=$ "never true" to $6=$ "always true", participants rate processes of experiential avoidance and psychological inflexibility. Reliability of the FAH-II is good $(\alpha=.84)$ [46].

In addition to the FAH-II, the German version of Chronic Pain Acceptance Questionnaire was used (CPAQ-D) [47]. On a 7-point scale that ranges from $0=$ "never true" to 6 "always true", participants rate their activity engagement (CPAQ-AE) and pain willingness (CPAQ-PW) on 20 items. Reliability of the German CPAQ and its subscales is good ( $\alpha=.84$ to .87$)$ [47].

\section{Analyses}

Analyses were divided in three parts: First, confirmatory factor analysis was used to evaluate the latent structure 
of the CAQ-G. Second, Mokken Scale Analysis was conducted to further evaluate the scalability and the adequateness of sum scores of the CAQ-G and CAQ-8-G. In a third step correlations between the short version, the long version, acceptance (FAH-II, CPAQ), depression (PHQ-9), anxiety (GAD-7), pain interference (MPI, BPI), pain intensity (NRS), and quality of life (SF12) were calculated.

\section{Confirmatory factor analysis: construct validity}

To examine the latent structure of the 18 items of the CAQ, a confirmatory factor analysis (CFA) was used. Based on the theoretical background and the results of the initial CAQ and the 8 item CAQ [25, 26, 48, 49] three competing models were derived. The first is a single factor model, representing a general committed action (CA) construct. The second is a two factor model representing (co)variances of positive or negative keyed items. Correlation between the two factors was allowed. The third is a bifactor model including three latent factors, a general factor representing the CA construct as in model 1 and two item class specific factors accounting for (co)variance of positive and negative keyed items, respectively. Correlations between factors were set to zero in the bifactor model. Values of negative keyed items were reversed for analyses.

Model fit of the three models were determined by Chi-square statistic. Due to the high sensitivity of chisquare, additional fit indices (RMSEA as a non-centrality parameter, SRMR as a residual fit index and CFI and TLI as incremental indices) were used if significance occurs. Based on standard criteria for model fit a RMSEA $\leq .05$, a CFI $>.95$, a TLI $>.95$ and SRMR $\leq .06$ indicates a good model fit [50].

A check for multivariate normality revealed that neither multivariate normality nor normality for single item was given. Thus, and to be consistent with later Mokken Scale Analysis (MSA) multivariate normality assumption and assumption of interval scale level of items were rejected. Instead of interval level, ordinal scale level was assumed. In case of non-normal ordered data diagonal weighted least square (DWLS) estimator is the most accurate estimator $[51,52]$ and therefore was used in CFA.

Convergent validity Convergent validity will be assessed via correlations to other constructs/ questionnaires. Medium absolute correlations (> I.5I) to psychological flexibility, acceptance and small to medium absolute correlation (>I.2I) with emotional and physical functioning would indicate good validity.

\section{Mokken scale analysis}

As done in the development of the original CAQ-8 [26] Mokken Scale Analysis (MSA) was conducted to evaluate the CAQ-8-G. In short, MSA is a scaling technique for ordinal data. Frequently, MSA is used for scaling test and it is related to nonparametric item response theory (IRT) [53]. MSA allows both, selecting items for scales and testing of assumptions of nonparametric IRT [53].

The key parameter in MSA is Loevinger's $\mathrm{H}$ - where the scaling parameter for item $\mathrm{i}$ is $\mathrm{H}_{\mathrm{i}}$ and the overall scalability of all items clustering onto scale $\mathrm{k}$ is $\mathrm{H}_{\mathrm{k}}$. $\mathrm{H}_{\mathrm{i}}$ indicates the strength of the relationship between a latent variable (committed action in this case) and item $i$. High scalability indicates whether the probability to score higher on item i increases with an increase on the latent variable. As a rule of thumb, a scale is considered weak if $\mathrm{H}<.4$, moderate if $.4 \leq \mathrm{H} \leq .5$ and strong if $\mathrm{H}>.5$ [54]. For detailed introduction see [53, 55, 56].

MSA assumes item monotonicity. Monotonicity was evaluated using item-rest regression [54, 57]. crit statistic was used to check for meaningful violations (crit $>40)$ [57, 58]. Further non-intersection is assumed. Nonintersection was evaluated by the restscore method (no violation: crit < 40, minor violations: crit $40-80$, serious violations: crit $>80)[53,55,57,58]$.

\section{Reliability}

As recommended by van der Ark [55] reliability of scales will be assessed by Cronbach's alpha [59], MolenaarSijtsma method (MS) [60, 61], lambda-2 [62] and latent class reliability coefficient (LCRC) [63]. In addition, we calculated omega $(\omega)$, since it provides a more unbiased estimation of reliability than the widely used Cronbach's alpha [64-66]. Bootstrapped and bias corrected confidence intervals was obtained for $\omega$ using the procedure introduced by Zhang and Yuan [67].

\section{Analysis software}

For all analyses, we used the software R [68]. For CFA the R package "lavaan" (version: 0.5-23.1097) was used [69]. MSA was conducted with the R package "mokken" $[53,55]$. Correlations were calculated using the "psych" package (version: 1.7.8.) [70]. For omega the "coefficientalpha" package was used [67]. The $\mathrm{R}$ script used for analysis and the anonymized data set can be requested from corresponding author (see availability of data and materials for further details).

\section{Results}

\section{Missingness and missing data handling}

Missingness only occurred in the descriptive variable pain duration. A total of 6 responses (3.3\%) were missing. For the calculation of pain duration missing values were excluded. 


\section{CFA: construct validity}

Three competing models were tested. First, a one factor model (all items only measure the construct Committed Action [CA]), second a two factor model (items measure one of two factors depending on how items are keyed) and third a bifactor model (CA factor plus two item class specific factors). Only the bifactor model resulted in an acceptable fit based on RMSEA, SRMR, CFI and TLI (see Table 2).

The construct validity is supported by the bifactor model. The fit of the bifactor model indicates that items measure a common latent construct (CA) as well as item class specific factors (=methodological factors of positive and negative keyed items). Loadings on the CA factor in the bifactor are presented in Table 3.

\section{Mokken scale analysis}

MSA for the CAQ-G resulted in a poor scalability $(\mathrm{H}=$ .391 [se $=.031]$ ). Assumption of monotonicity was met, while non-intersection was highly violated (12 minor and 6 critical violations). Reliability was high $(\alpha=.91$ [95\% CI: .89 to .93$], \mathrm{MS}=.91$, lambda. $2=.92, \mathrm{LCRC}=$ $.91, \omega=.91$ [95\% CI: .88 to .93]). Scalability for all positive items was strong $(\mathrm{H}=.643[\mathrm{se}=.039])$. Monotonicity was met and evaluation of non-intersection yielded only two minor violation $\left(\right.$ crit $_{\text {item } 4}=.40$, crit $\left._{\text {item } 15}=43\right)$. Reliability of the positive subscale was excellent $(\alpha=.93$ [95\% CI: .92 to .95 ), $\mathrm{MS}=.94$, lambda. $2=.93, \mathrm{LCRC}=$ $.92, \omega=.93$ [95\% CI: .91 to .95$]$ ). Negative subscale showed poor scalability $(\mathrm{H}=.365[\mathrm{se}=.033])$, no violations of monotonicity and four minor and one serious violation of non-intersection. Reliability was good $(\alpha=$ .82 [95\% CI: .79 to .86$], \mathrm{MS}=.83$, lambda. $2=.83$, LCRC $=.83, \omega=.83$ [95\% CI: .78 to .86$]$ ).

The CAQ-8-G, a scale with the corresponding items of the English CAQ-8 was tested with MSA in addition to the CAQ-G. This scale was medium to strong $(\mathrm{H}=$ .453 [se $=.038])$ with high reliability $(\alpha=.85$ [95\% CI: .82 to .89 ), $\mathrm{MS}=.86$, lambda. $2=.86, \mathrm{LCRC}=.86, \omega=.85$ [95\% CI: .79 to .88]). Assumption of monotonicity was met. Seven items showed minor violations of nonintersection.

\section{Convergent validity}

To assess convergent validity of the scale, correlations with other measures were calculated. As assumed the
CAQ-G has good convergent validity: The CAQ-G scales demonstrated high correlations with other measures of psychological flexibility or facets of psychological flexibility (CPAQ and FAH). Further, medium correlations to indicators for physical and emotional functioning (MPI-interference, BPI-interference, PHQ, GAD) as well as to quality of life (SF12 MCS) were found. No correlation with pain intensity (NRS) and SF12 PCS was found. See Table 4 for correlations.

\section{Discussion}

Aim of this study was to validate the CAQ-G and CAQ8-G to provide a comprehensive assessment of committed action (CA) in German. Overall, the findings within this study are similar to the results of the English CAQ and CAQ-8 $[25,26]$. Construct validity of the CAQ-G was demonstrated by the bifactor model. Expectations regarding convergent validity were met. Reliability was high. While MSA for the positive subscale of the CAQ$\mathrm{G}$ and the CAQ-8-G indicate that the use of sum scores is adequate, sum score of the negative subscale should be used with caution due to major violations.

As shown in the validation of the original CAQ and assumed for the CAQ-G, a bifactor model explains the latent structure of the CAQ best $[25,26]$. Given the theoretical background of the CAQ it can be assumed that the general factor represents the latent construct committed action, while the other factors explain covariance introduced by methodology (e.g. positive and negative wording of items). While the construct validity is demonstrated by the present results, it also has to be highlighted that also two-factor model was fitting the original CAQ [26]. This is not true for the CAQ-G, since the RMSEA exceeded the pre-defined cut-off. Future studies using the CAQ-G should investigate whether this is an artifact of the present study or a robust finding. However, based on the present findings only a the bifactor model confirms the construct validity of the CAQ-G.

While CFA confirms the assumed structure, it also highlights that some items are poor or no meaningful indicators for the general latent factor CA (e.g. item 05: loading $=.13, p=.472$ ) and only good indicators for the methodological factors. Unsurprisingly MSA shows that the scalability of the total scale including the poor indicators is weak. Thus, a short version only including strong indicators would offer a more reliable assessment

Table 2 Confirmatory factor analysis - comparison of models

\begin{tabular}{lllllllll}
\hline & \multicolumn{1}{l}{ Noncentrality parameters } \\
\cline { 2 - 9 } Modell & Chi-square & $\mathrm{df}$ & $p$ value & RMSEA & $90 \% \mathrm{Cl}$ & SRMR & \multicolumn{2}{l}{ Incremental indices } \\
\hline One factor & 791.37 & 135 & $<.001$ & .16 & $.15-.18$ & .10 & .98 & .98 \\
Two factor & 332.78 & 134 & $<.001$ & .09 & $.08-.10$ & .06 & .99 & .99 \\
Bifactor & 173.22 & 117 & $<.001$ & .05 & $.04-.07$ & .05 & .99 & .99 \\
\hline
\end{tabular}


Table 3 Factor loadings on latent committed action variable based on bifactor model

\begin{tabular}{llll}
\hline Item & Standardized loading on CA & $P$ value $^{1}$ & Included in short scale $^{\prime}$ \\
\hline 01 & 0.711 & $<.001$ & \\
02 & 0.941 & $<.001$ & $\checkmark$ \\
03 & 0.950 & $<.001$ & $\checkmark$ \\
04 & 0.897 & $<.001$ & $\checkmark$ \\
$05^{n}$ & 0.126 & .472 & \\
06 & 0.811 & $<.001$ & \\
07 & 0.876 & $<.001$ & $\checkmark$ \\
$08^{n}$ & 0.612 & $<.001$ & $\checkmark$ \\
$09^{n}$ & 0.436 & $<.001$ & $\checkmark$ \\
$10^{n}$ & 0.447 & $<.001$ & \\
$11^{n}$ & 0.349 & .023 & \\
12 & 0.670 & .014 & \\
13 & 0.693 & .004 & \\
$14^{n}$ & 0.453 & $<.001$ & \\
15 & 0.656 & $<.001$ & \\
$16^{n}$ & 0.412 & $<.001$ & \\
$17^{n}$ & 0.504 & $<.001$ & $\checkmark$ \\
$18^{n}$ & 0.484 & $<.001$ & $\checkmark$ \\
\hline
\end{tabular}

Note: ${ }^{1} p$-values were obtained using bootstrap; ${ }^{\mathrm{n}}$ indicates negative keyed items; of CA. MSA demonstrates that the CAQ-8-G is such a scale. Similar to the original CAQ-8 [26], the CAQ-8-G showed medium to strong scalability and excellent reliability. This is also true for the positive subscale of the CAQ, but not for the negative subscale. Hence, only the CAQ-8-G or the positive subscale of the CAQ should be used in practice.

In terms of convergent validity, the present results almost perfectly mirror the results from the original CAQ validation and the hypothesis regarding the correlations were met. Especially, The correlations of the CAQ-G and CAQ-8-G with variables of psychological flexibility, emotional and physical functioning are almost identical to the correlations of the English versions $\left(\mathrm{r}_{\mathrm{CPAQ}}=.49\right.$, $\mathrm{r}_{\mathrm{PHQ}-9}=-.57, \mathrm{r}_{\mathrm{SF}-36-\text { Mental }}=.58$ ) [25]. Overall, the medium correlations between psychological flexibility, facets of psychological flexibility, emotional and physical functioning are in line with many prior studies [25, 2732]. Correlations with variables of emotional and physical functioning as well as with quality of life indicate that psychological flexibility as wider process, or committed action specifically, might act as mechanisms of change in treatments of chronic pain. As such measures of these processes may help in the further development of psychological treatments for chronic pain.

Although the present results are promising, a few limitations have to be taken into account for interpretation. All analyses are based on a single sample of 181 German individuals with chronic pain from an RCT evaluating the

Table 4 Correlation analysis: convergent validity

\begin{tabular}{|c|c|c|c|c|c|c|c|c|c|c|c|c|c|c|c|}
\hline & 1 & 2 & 3 & 4 & 5 & 6 & 7 & 8 & 9 & 10 & 11 & 12 & 13 & 14 & $\overline{15}$ \\
\hline$\overline{1 \mathrm{CPAQ}_{\mathrm{AE}}}$ & $1^{*}$ & & & & & & & & & & & & & & \\
\hline $2 \mathrm{CPAQ}_{\mathrm{PW}}$ & $.59^{*}$ & $1^{*}$ & & & & & & & & & & & & & \\
\hline $3 \mathrm{CPAQ}_{\text {total }}$ & $.93^{*}$ & $.85^{*}$ & $1^{*}$ & & & & & & & & & & & & \\
\hline 4 FAH II & $-.59^{*}$ & $-.65^{*}$ & $-.69^{*}$ & $1^{*}$ & & & & & & & & & & & \\
\hline $5 \mathrm{MPI}$ - interference & $-.63^{*}$ & $-.46^{*}$ & $-.63^{*}$ & $.45^{*}$ & $1^{*}$ & & & & & & & & & & \\
\hline $6 \mathrm{BPI}$ - interference & $-.51^{*}$ & $-.4^{*}$ & $-.52^{*}$ & $.43^{*}$ & $.76^{*}$ & $1^{*}$ & & & & & & & & & \\
\hline $7 \mathrm{PHQ}$ & $-.5^{*}$ & $-.49^{*}$ & $-.55^{*}$ & $.64^{*}$ & $.58^{*}$ & $.61^{*}$ & $1^{*}$ & & & & & & & & \\
\hline $8 \mathrm{GAD}$ & $-.39^{*}$ & $-.48^{*}$ & $-.48^{*}$ & $.68^{*}$ & $.39^{*}$ & $.42^{*}$ & $.78^{*}$ & $1^{*}$ & & & & & & & \\
\hline 9 NRS & $-.38^{*}$ & $-.23^{*}$ & $-.35^{*}$ & $.22^{*}$ & $.73^{*}$ & $.77^{*}$ & $.43^{*}$ & $.22^{*}$ & $1^{*}$ & & & & & & \\
\hline $10 \mathrm{SF} 12_{\mathrm{PCS}}$ & $.34^{*}$ & .19 & $.31^{*}$ & -.05 & $-.54^{*}$ & $-.48^{*}$ & -.2 & .01 & $-.55^{*}$ & $1^{*}$ & & & & & \\
\hline $11 \mathrm{SF} 12_{\mathrm{MCS}}$ & $.53^{*}$ & $.5^{*}$ & $.58^{*}$ & $-.76^{*}$ & $-.47^{*}$ & $-.45^{*}$ & $-.77^{*}$ & $-.74^{*}$ & $-.22^{*}$ & -.12 & $1^{*}$ & & & & \\
\hline 12 CAQ-8-G & $.54^{*}$ & $.48^{*}$ & $.58^{*}$ & $-.67^{*}$ & $-.31^{*}$ & $-.28^{*}$ & $-.48^{*}$ & $-.49^{*}$ & -.12 & .02 & $.57^{*}$ & $1^{*}$ & & & \\
\hline $13 C A Q-G_{\mathrm{Neg}}$ & $.39^{*}$ & $.43^{*}$ & $.45^{*}$ & $-.63^{*}$ & $-.22^{*}$ & -.2 & $-.38^{*}$ & $-.48^{*}$ & -.04 & .01 & $.48^{*}$ & $.84^{*}$ & $1^{*}$ & & \\
\hline $14 \mathrm{CAQ}-\mathrm{G}_{\mathrm{Pos}}$ & $.54^{*}$ & $.46^{*}$ & $.56^{*}$ & $-.56^{*}$ & $-.30^{*}$ & $-.28^{*}$ & $-.43^{*}$ & $-.40^{*}$ & -.11 & .02 & $.53^{*}$ & $.86^{*}$ & $.55^{*}$ & $1^{*}$ & \\
\hline 15 CAQ-G & $.53^{*}$ & $.51^{*}$ & $.58^{*}$ & $-.67^{*}$ & $-.30^{*}$ & $-.28^{*}$ & $-.46^{*}$ & $-.50^{*}$ & -.09 & .02 & $.57^{*}$ & $.96^{*}$ & $.87^{*}$ & $.89^{*}$ & $1^{*}$ \\
\hline
\end{tabular}

Note: ${ }^{*}$ indicates significance below $p \leq .05$ (adjusted for multiple testing); MPI Multidimensional Pain Inventory, BPI Brief Pain Inventory, PHQ 9 Patient Health Questionnaire (9 items version), GAD 7 Generalized Anxiety Disorder questionnaire (7 item version), NRS Numeric Rating Scale of Pain Intensity, SF12_PCS Health related quality of life the Short Form 12 (physical), SF12 MCS Health related quality of life the Short Form 12 (mental health), FHA // German version of the Acceptance and Action Questionnaire - II (AAQ-II), low values indicate high flexibility, CPAQ AE Chronic Pain Acceptance Questionnaire activity engagement scale, CPAQ PW Chronic Pain Acceptance Questionnaire pain willingness scale, CPAQ Total Chronic Pain Acceptance Questionnaire, CAQ-G German version of the committed action questionnaire, $C A Q-G_{\text {neg }}$ includes only negative keyed items, $C A Q-G_{\text {pos }}$ includes only positive keyed items, $C A Q-8-G$ German version of the English short version of the CAQ 
effectiveness of an internet-based intervention [33, 34]. Although individuals show a variety in socio-demographic backgrounds and in pain related variables such as pain duration of pain location, the present sample is not a normative sample representative for the general population. Hence, it is unclear whether similar results would emerge in other pain populations, population with different somatic or mental health conditions, or healthy populations. Thus, the CAQ-G and CAQ-8-G should be validated in additional populations to examine generalizability.

Moreover, the reduced items in the CAQ-8-G might lead to loss of information and the construct of CA is less comprehensively covered. However, the high correlation between the CAQ-G and the CAQ-8-G $(r=.96)$ indicate a rather low and maybe insignificant loss of information. Thus, the gains of time efficiency and loss of information should be weighed for each individual study.

Lastly a limitation emerges of the applied definition of committed action itself. As highlighted by McCracken and colleagues [26] the definition of committed action applied in the English CAQ and CAQ-8 and the respective German versions may seem somewhat narrow: For instance, behavior change with no difficulties or integration of behavior change into generalized patterns are not covered by the CAQ. Future studies might target this limitation and expand the current definition by developing new items. However, based on the high and medium correlations to health and psychological flexibility constructs it can be assumed that the current CAQ already covers relevant aspects of committed action.

\section{Conclusion}

With the development and examination of the CAQ-G and CAQ-8-G the present study provides a new way to assess patterns of actions and behavior in German populations. Construct validity and convergent validity of the CAQ-G and the CAQ-8-G was good. However, taking the scalability into account, only the CAQ-8-G and the positive subscale of the CAQ-G should be used for a valid and reliable assessment of CA in German populations. Furthermore, the present results highlight the close relationship between psychological flexibility and health. To develop a deeper understanding of the mechanisms behind effective therapy and the causal relationships between CA and health, further studies are needed.

\section{Supplementary information}

Supplementary information accompanies this paper at https://doi.org/10. 1186/s12955-020-01497-8.

Additional file 1. Committed Action Questionnaire.

\section{Abbreviations}

ACT: Acceptance and Commitment Therapy; CAQ: Committed Actions Questionnaire; CAQ-8: Short version of Committed Actions Questionnaire;
IRT: Item Response Theory; MSA: Mokken Scaling Analysis; RCT: Randomized Controlled Trial; DRKS: German Clinical Trial Register; CAQ-G: German Committed Action Questionnaire; CAQ-8-G: Short version of German Committed Actions Questionnaire; IMMPACT: Initiative on Methods, Measurement, and Pain Assessment in Clinical Trials; MPI: Multidimensional Pain Inventory; BPI: Brief Pain Inventory; PHQ-9: Patient Health Questionnaire; GAD-7: Generalized Anxiety Disorder questionnaire; SF12: Health-related quality of life Short Form 12; SF12 PCS: Divided into physical; SF12 MCS: Mental health; AAQ-II: Acceptance and Action Questionnaire — II; FAHII: Fragebogen zu Akzeptanz und Handeln II; CPAQ-D: Chronic Pain Acceptance Questionnaire; CPAQ-AE: Activity Engagement; CPAQ-PW: Pain Willingness; NRS: Numerical Rating Scale; CFA: Confirmatory Factor Analysis; CA): Committed Action; DWLS: Diagonal Weighted Least Square; MS: Molenaar-Sijtsma method; LCRC: Latent Class Reliability Coefficient

\section{Acknowledgements}

We are grateful to Mr. Peter Spaeth for his contributions during the measure translation.

\section{Authors' contributions}

All authors substantially contributed to the present study. YT conducted the data analyses and prepared the first draft of the manuscript. JL was responsible for the data collection in the ACTonPain study. JL, LM and HB were responsible for the design of this study and for the translation. All authors agreed to be accountable for all aspects of the work in ensuring that questions related to the accuracy or integrity of any part of the work are appropriately investigated and resolved. The author(s) read and approved the final manuscript

\section{Funding}

Investigator initiated and self-funded study. Open access funding provided by Projekt DEAL.

\section{Availability of data and materials}

The dataset analyzed during the current study is not publicly available due to the sensitive nature of patients' data. Participants agreed on the use of their anonymized data for research purposes only. An anonymized version of the dataset with no direct or indirect identifiers can be provided by HB on reasonable request, in which clear research and no other purposes are stated. The R script used for all analyses can be provided on request by YT. Data requestors will need to sign a data access agreement. Provision of data is subject to data security regulations. Investigator support depends on available resources.

\section{Ethics approval and consent to participate}

All procedures in the study ACTonPain [33, 34], in which the present data was collected, were approved by the ethics committee at the AlbertLudwigs-University of Freiburg (Nr.: 387/14). The ACTonPain trial was registered at the German Clinical Trial Register (DRKS): DRKS00006183. Only eligible participants who provided an informed consent were included in the study.

\section{Consent for publication}

All authors read and approved the final version of this manuscript.

\section{Competing interests}

The authors declare that they have no competing interests.

\section{Author details}

'Department of Research Methods, Institute of Psychology and Education, Ulm University, Albert-Einstein-Allee 47, 89069 Ulm, Germany. ${ }^{2}$ Department of Clinical Psychology and Psychotherapy, Institute of Psychology and Education, Ulm University, Lise-Meitner-Str. 16, 89081 Ulm, Germany. ${ }^{3}$ Department of Psychology, Uppsala University, Box 1225751 42, Uppsala, Sweden. ${ }^{4}$ Institute of Sport and Sport Science, University Freiburg, Schwarzwaldstraße 175, 79117 Freiburg, Germany. 
Received: 18 September 2019 Accepted: 16 July 2020

\section{Published online: 03 August 2020}

\section{References}

1. Kashdan TB, Rottenberg J. Psychological flexibility as a fundamental aspect of health. Clin Psychol Rev. 2010;30:865-78. https://doi.org/10.1016/j.cpr. 2010.03.001.

2. Hayes SC, Luoma JB, Bond FW, Masuda A, Lillis J. Acceptance and commitment therapy: model, processes and outcomes. Behav Res Ther. 2006; $44: 1-25$.

3. Masuda A, Tully EC. The role of mindfulness and psychological flexibility in somatization, depression, anxiety, and general psychological distress in a nonclinical college sample. J Evid Based Complementary Altern Med. 2012 17:66-71. https://doi.org/10.1177/2156587211423400.

4. Hayes SC, Strosahl K, Wilson KG. Acceptance and commitment therapy : the process and practice of mindful change. New York: Guilford Press; 2012. https://www.guilford.com/books/Acceptance-and-Commitment-Therapy/ Hayes-Strosahl-Wilson/9781462528943.

5. Lin J, Klatt L-I, McCracken LM, Baumeister H. Psychological flexibility mediates the effect of an online-based acceptance and commitment therapy for chronic pain. Pain. 2018;159:663-72. https://doi.org/10.1097/j. pain.0000000000001134

6. Gentili C, Rickardsson J, Zetterqvist V, Simons LE, Lekander M, Wicksell RK. Psychological flexibility as a resilience factor in individuals with chronic pain. Front Psychol. 2019;10:2016. https://doi.org/10.3389/fpsyg.2019.02016.

7. Veehof MM, Oskam M-J, Schreurs KMG, Bohlmeijer ET. Acceptance-based interventions for the treatment of chronic pain: a systematic review and meta-analysis. Pain. 2011;152:533-42. https://doi.org/10.1016/j.pain.2010.11. 002.

8. Veehof MM, Trompetter H, Bohlmeijer ET, Schreurs KMG. Acceptance- and mindfulness-based interventions for the treatment of chronic pain: a metaanalytic review. Cogn Behav Ther. 2016;45:5-31.

9. Brown $M$, Glendenning A, Hoon AE, John A. Effectiveness of web-delivered acceptance and commitment therapy in relation to mental health and wellbeing: a systematic review and meta-analysis. J Med Internet Res. 2016;18: e221. https://doi.org/10.2196/jmir.6200.

10. Twohig MP, Levin ME. Acceptance and commitment therapy as a treatment for anxiety and depression: a review. Psychiatr Clin North Am. 2017;40:75170

11. Kazdin AE. Mediators and mechanisms of change in psychotherapy research. Annu Rev Clin Psychol. 2007;3:1-27. https://doi.org/10.1146/ annurev.clinpsy.3.022806.091432.

12. Cuijpers $P$. The future of psychotherapy research: stop the waste and focus on issues that matter. Epidemiol Psychiatr Sci. 2016;25:291-4.

13. Murphy R, Cooper Z, Hollon SD, Fairburn CG. How do psychological treatments work? Investigating mediators of change. Behav Res Ther. 2009; 47:1-5. https://doi.org/10.1016/j.brat.2008.10.001.

14. Probst T, Baumeister H, McCracken L, Lin J, Probst T, Baumeister $H$, et al. Baseline psychological inflexibility moderates the outcome pain interference in a randomized controlled trial on internet-based acceptance and commitment therapy for chronic pain. J Clin Med. 2018;8:24. https://doi.org/ 10.3390/jcm8010024

15. Vowles KE, McCracken LM, McLeod C, Eccleston C. The chronic pain acceptance questionnaire: confirmatory factor analysis and identification of patient subgroups. Pain. 2008;140:284-91.

16. Brown KW, Ryan RM. The benefits of being present: mindfulness and its role in psychological well-being. J Pers Soc Psychol. 2003;84:822-48.

17. Wicksell RK, Lekander M, Sorjonen K, Olsson GL. The Psychological Inflexibility in Pain Scale (PIPS)--statistical properties and model fit of an instrument to assess change processes in pain related disability. Eur J Pain. 2010;14:771.e1-14.

18. Yu L, Norton S, McCracken LM. Change in "self-as-context" ("perspectivetaking") occurs in acceptance and commitment therapy for people with chronic pain and is associated with improved functioning. J Pain. 2017;18: 664-72.

19. Yu L, McCracken LM, Norton S. The self experiences questionnaire (SEQ): preliminary analyses for a measure of self in people with chronic pain. $J$ Contextual Behav Sci. 2016;5:127-33. https://doi.org/10.1016/j.jcbs.2016.07. 006.

20. McCracken LM, Yang S-Y. The role of values in a contextual cognitivebehavioral approach to chronic pain. Pain. 2006;123:137-45.
21. Baranoff J, Hanrahan SJ, Kapur D, Connor JP. Acceptance as a process variable in relation to catastrophizing in multidisciplinary pain treatment. Eur J Pain. 2013;17:101-10.

22. Vowles KE, Wetherell JL, Sorrell JT. Targeting acceptance, mindfulness, and values-based action in chronic pain: findings of two preliminary trials of an outpatient group-based intervention. Cogn Behav Pract. 2009;16:49-58. https://doi.org/10.1016/j.cbpra.2008.08.001

23. Vowles KE, Witkiewitz K, Sowden G, Ashworth J. Acceptance and commitment therapy for chronic pain: evidence of mediation and clinically significant change following an abbreviated interdisciplinary program of rehabilitation. J Pain. 2014;15:101-13. https://doi.org/10.1016/j.jpain.2013.10. 002.

24. Levin ME, Hildebrandt MJ, Lillis J, Hayes SC. The impact of treatment components suggested by the psychological flexibility model: a metaanalysis of laboratory-based component studies. Behav Ther. 2012;43:74156. https://doi.org/10.1016/J.BETH.2012.05.003.

25. McCracken LM. Committed action: an application of the psychological flexibility model to activity patterns in chronic pain. J Pain. 2013;14:828-35. https://doi.org/10.1016/j.jpain.2013.02.009.

26. McCracken LM, Chilcot J, Norton S. Further development in the assessment of psychological flexibility: A shortened Committed Action Questionnaire (CAQ-8). Eur J Pain (United Kingdom). 2015;19:677-85.

27. Huijnenl IPJ, Verbunt JA, PetersI ML, Smeetsl RJEM, Kindermansl HPJ, Roelofs $J$, et al. Differences in activity-related behaviour among patients with chronic low back pain. Eur J Pain. 2011;15:748-55. https://doi.org/10.1016/j. ejpain.2010.11.015

28. Kindermans HPJ, Roelofs J, Goossens MEJB, Huijnen IPJ, Verbunt JA, Vlaeyen JWS. Activity patterns in chronic pain: underlying dimensions and associations with disability and depressed mood. J Pain. 2011;12:1049-58. https://doi.org/10.1016/j.jpain.2011.04.009.

29. McCracken LM, Samuel VM. The role of avoidance, pacing, and other activity patterns in chronic pain. Pain. 2007;130:119-25. https://doi.org/10. 1016/j.pain.2006.11.016.

30. Vlaeyen JWS, Linton SJ. Fear-avoidance and its consequences in chronic musculoskeletal pain: a state of the art. Pain. 2000;85:317-32 http://www. ncbi.nlm.nih.gov/pubmed/10781906. Accessed 1 Dec 2017.

31. Wicksell RK, Renöfält J, Olsson GL, Bond FW, Melin L. Avoidance and cognitive fusion - central components in pain related disability? Development and preliminary validation of the psychological inflexibility in pain scale (PIPS). Eur J Pain. 2008;12:491-500. https://doi.org/10.1016/j. ejpain.2007.08.003.

32. Scott W, McCracken LM. Psychological flexibility, acceptance and commitment therapy, and chronic pain. Curr Opin Psychol. 2015;2:91-6. https://doi.org/10.1016/j.copsyc.2014.12.013.

33. Lin J, Lüking M, Ebert DD, Buhrman M, Andersson G, Baumeister $\mathrm{H}$. Effectiveness and cost-effectiveness of a guided and unguided internetbased acceptance and commitment therapy for chronic pain: study protocol for a three-armed randomised controlled trial. Internet Interv. 2015; 2:7-16. https://doi.org/10.1016/j.invent.2014.11.005.

34. Lin J, Paganini S, Sander L, Lüking M, Ebert DD, Buhrman M, et al. An internet-based intervention for chronic pain. Dtsch Arztebl Int. 2017;114 681-8. https://doi.org/10.3238/arztebl.2017.0681.

35. Beaton DE, Bombardier C, Guillemin F, Ferraz MB. Guidelines for the process of cross-cultural adaptation of self-report measures. Spine (Phila Pa 1976). 2000:25:3186-91.

36. Dworkin RH, Turk DC, Farrar JT, Haythornthwaite JA, Jensen MP, Katz NP, et al. Core outcome measures for chronic pain clinical trials: IMMPAC recommendations. Pain. 2005;113:9-19.

37. Dworkin RH, Turk DC, Wyrwich KW, Beaton D, Cleeland CS, Farrar JT, et al. Interpreting the clinical importance of treatment outcomes in chronic pain clinical trials: IMMPACT recommendations. J Pain. 2008;9:105-21.

38. Flor H, Rudy TE, Birbaumer N, Streit B, Schugens MM. Zur Anwendbarkeit des West Haven-Yale Multidimensional Pain Inventory im deutschen Sprachraum - Daten zur Reliabilität und Validität des MPI-D. Schmerz. 1990; 4:82-7.

39. Radbruch L, Loick G, Kiencke P, Lindena G, Sabatowski R, Grond S, et al. Validation of the German version of the brief pain inventory. J Pain Symptom Manag. 1999:18:180-7.

40. Gräfe K, Zipfel S, Herzog W, Löwe B. Screening psychischer störungen mit dem "Gesundheitsfragebogen für Patienten (PHQ-D)". Ergebnisse der Deutschen validierungsstudie. Diagnostica. 2004;50:171-81. 
41. Löwe B, Decker O, Müller S, Brähler E, Schellberg D, Herzog W, et al. Validation and standardization of the generalized anxiety disorder screener (GAD-7) in the general population. Med Care. 2008;46:266-74 https:// insights.ovid.com/pubmed?pmid=18388841. Accessed 16 Sep 2017.

42. Spitzer RL, Kroenke K, Williams JB, Löwe B. A brief measure for assessing generalized anxiety disorder. Arch Intern Med. 2006;166:1092-7.

43. Rief W, Nanke A, Klaiberg A, Braehler E. Base rates for panic and depression according to the brief patient health questionnaire: a population-based study. J Affect Disord. 2004;82:271-6.

44. Luo X, Lynn George M, Kakouras I, Edwards CL, Pietrobon R, Richardson W, et al. Reliability, Validity, and Responsiveness of the Short Form 12-Item Survey (SF-12) in Patients With Back Pain. Spine (Phila Pa 1976). 2003;28: 1739-45. https://doi.org/10.1097/01.BRS.0000083169.58671.96.

45. Bond FW, Hayes SC, Baer RA, Carpenter KM, Guenole N, Orcutt HK, et al. Preliminary psychometric properties of the acceptance and action questionnaireII: a revised measure of psychological inflexibility and experiential avoidance. Behav Ther. 2011;42:676-88. https://doi.org/10.1016/j.beth.2011.03.007.

46. Hoyer J, Gloster AT. Psychologische Flexibilität messen: Der Fragebogen zu Akzeptanz und Handeln II. Verhaltenstherapie. 2013;23:42-4.

47. Nilges P, Köster B, Schmidt CO. Schmerzakzeptanz - Konzept und Überprüfung einer deutschen Fassung des Chronic Aain Acceptance Questionnaire. Schmerz. 2007;21:57-67.

48. Bailey RW, Vowles KE, Witkiewitz K, Sowden G, Ashworth J. Examining committed action in chronic pain: further validation and clinical utility of the committed action questionnaire. J Pain. 2016;17(10):1095-104.

49. Åkerblom S, Perrin S, Fischer MR, McCracken LM. A validation and generality study of the committed action questionnaire in a Swedish sample with chronic pain. Int J Behav Med. 2016;23:260-70. https://doi.org/10.1007/ s12529-016-9539-X.

50. Hu LT, Bentler PM. Cutoff criteria for fit indexes in covariance structure analysis: conventional criteria versus new alternatives. Struct Equ Model. 1999;6:1-55

51. Mîndrilã D. Maximum likelihood (ML) and diagonally wighted least squares (DWLS) estimation procedures: a comparison of estimation bias with ordinal and multivariate non-normal data. Int J Digit Soc. 2010;1:60-6.

52. $\mathrm{Li} \mathrm{C}-\mathrm{H}$. The performance of ML, DWLS, and ULS estimation with robust corrections in structural equation models with ordinal variables. Psychol Methods. 2016;21:369-87. https://doi.org/10.1037/met0000093.

53. van der Ark LA. Mokken scale analysis in R. J Stat Softw. 2007;20:183-208. https://doi.org/10.1007/s11336-007-9034-z.

54. Mokken RJ. A theory and procedure of scale analysis : with applications in political research. Mouton; 1971.

55. van der Ark LA. New Developments in Mokken Scale Analysis in R. 2012;48: 1-27 https://www.jstatsoft.org/article/view/v048i05.

56. Sijtsma K, van der Ark LA. A tutorial on how to do a Mokken scale analysis on your test and questionnaire data. Br J Math Stat Psychol. 2017;70:137-58.

57. Chou YH, Lee CP, Liu CY, Hung Cl. Construct validity of the depression and somatic symptoms scale: evaluation by Mokken scale analysis. Neuropsychiatr Dis Treat. 2017;13:205-11.

58. Molenaar IW, Sijtsma K. User's manual MSP5 for windows. Software manual. IEC ProGAMMA: Groningen; 2000

59. Cronbach $L$. Coefficient alpha and the internal structure of tests. Psychometrika. 1951;16:297-334. https://doi.org/10.1007/BF02310555.

60. Molenaar IW, Sijtsma K. Mokken's approach to reliability estimation extended to multicategory items. Kwant Methoden. 1988;9:115-26.

61. Sijtsma K, Molenaar IW. Reliability of test scores in nonparametric item response theory. Psychometrika. 1987;52:79-97 https://link.springer.com/ content/pdf/10.1007/BF02293957.pdf. Accessed 1 Dec 2017.

62. Guttman L. A basis for analyzing test-retest reliability. Psychometrika. 1945;10: 255-82 http://www.ncbi.nlm.nih.gov/pubmed/21007983. Accessed 1 Dec 2017.

63. van der Ark LA, van der Palm DW, Sijtsma K. A latent class approach to estimating test-score reliability. Appl Psychol Meas. 2011;35:380-92. https:// doi.org/10.1177/0146621610392911

64. McNeish D. Thanks coefficient alpha, we'll take it from here. Psychol Methods. 2018;23:412-33.

65. Dunn TJ, Baguley T, Brunsden V. From alpha to omega: a practical solution to the pervasive problem of internal consistency estimation. Br J Psychol. 2014;105:399-412. https://doi.org/10.1111/bjop.12046.

66. Revelle W, Zinbarg R. Coefficients alpha, Beta, omega and GLB: comments on Sijtsma. Psychometrika. 2009;74:145-54 http://personality-project.org/ revelle/publications/revelle.zinbarg.08.pdf. Accessed 25 Mar 2017.
67. Zhang Z, Yuan K. Robust Coefficients Alpha and Omega and Confidence Intervals With Outlying Observations and Missing Data : Methods and Software; 2016.

68. R Core Team. R: A Language and Environment for Statistical Computing, vol. 0. Vienna: R Foundation for Statistical Computing; 2018:\{ISBN\} 3-900051-070. doi:http://www.R-project.org/.

69. Rosseel Y. Lavaan: an R package for structural equation modeling. J Stat Softw. 2009;30:1-3.

70. Revelle W. psych: Procedures for Psychological, Psychometric, and Personality Research; 2018

\section{Publisher's Note}

Springer Nature remains neutral with regard to jurisdictional claims in published maps and institutional affiliations.
Ready to submit your research? Choose BMC and benefit from:

- fast, convenient online submission

- thorough peer review by experienced researchers in your field

- rapid publication on acceptance

- support for research data, including large and complex data types

- gold Open Access which fosters wider collaboration and increased citations

- maximum visibility for your research: over $100 \mathrm{M}$ website views per year

At BMC, research is always in progress.

Learn more biomedcentral.com/submissions 